\title{
Conference Impressions
}

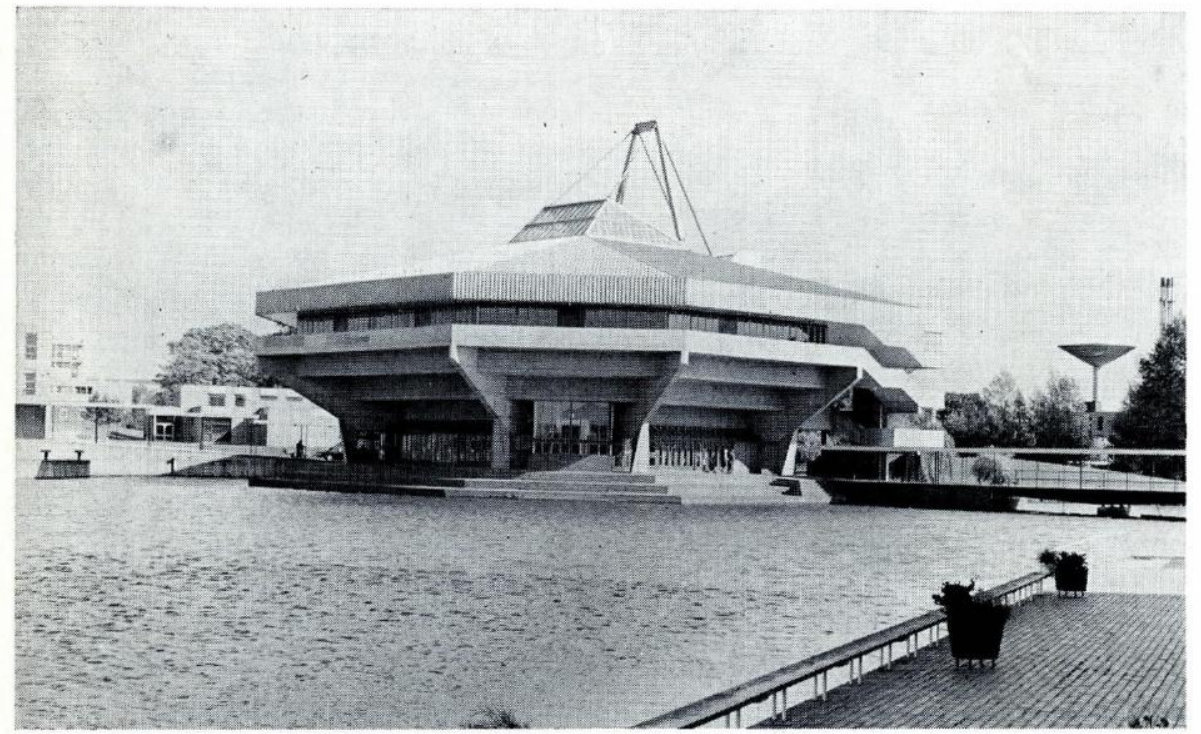

Some 700 physicists converged on the campus of York University for the 4th EPS General Conference that began on September 25. Low modern buildings distributed round a system of artificial lakes and streams (that made a virtue out of a drainage necessity) formed an attractive setting for the Conference, even if it tended to fragment the parallel symposia and make attendance at more than one, on any half-day, rather difficult. A varied programme had been prepared with plenary sessions alternating with eight specialized symposia ; in addition, an exhibition of equipment and publications was mounted and, tucked away rather out of reach, an area was set aside for posters.

\section{Physics in Society}

Physicists have the reputation of concentrating on their own specialized subject to the exclusion of all else, but a strong accent at York was put on interdisciplinarity and also on the practical and social implications of physics research and development. This is of particular importance when it is remembered that physics provides a background that is relevant to many fields. Physicists have a greater mobility than scientists from any other scientific discipline due to the rigorous training that the subject gives. As one of the Physics and Society Study Groups concluded, this means that the goal at school level should be to teach the methodology of physics rather than try and feed in encyclopaedic facts. Piaget sees physics as a subject that advances much as the individual advances, relating and comparing experience with conception. This remains valid after school days are over.

York was an opportunity to roam and listen, mix subjects and objectives alike. Volume $2 \mathrm{G}$ in the EPS Europhysics Conference Abstracts series provides a comprehensive summary of the invited and contributed papers and the Proceedings will do justice to the large amount of information unfolded during the Conference. Europhysics News makes no attempt to compete with either but instead, recalls some personal impressions gained at the meetings and the study groups, round the posters and exhibition, and in the corridors.

The outward looking tone of much of the Conference was set from the beginning with a plenary session entitled the Age of Silicon. Si is the substance best understood by man, implying equally that we are very conscious also of what we don't know about its characteristics, notably its surface. The application of Si technology has penetrated all our daily life and the revolution of microelectronics is expected to modify the lives of people as much as did the industrial revolution. The first field transistor appeared about 35 years ago and the enormous progress towards integrated circuits made since, has been brought about very largely by the mastery of defects. Less than 20 years ago, 10 components per chip was normal, by 1970 the figure had risen to several hundred, by today to tens of thousand and by (say) 1983 it will top the million. Apart from the enormous technological progress that has brought this about with the use of electron beams for mask fabrication
A mosaic of impressions carried away from the EPS 4th General Conference not necessarily the latest results, but the points of interest that made impact on an international group of participants drawn from Czechoslovakia, France, Federal Republic of Germany, Iran, Israel, Poland, Portugal, Switzerland and the U.K. The collaboration of speakers and session chairmen is gratefully acknowledged.

Central conference hall of York University seen from the physics buildings.

(and the prospect of using synchrotron radiation to increase the function density by another factor of 15) and X-rays instead of UV to expose the photo resist on the $\mathrm{Si}$, there has had to be a huge advance in conceptual design. Chips may be small, but the design of one today, requires as many drawings as does a nine storey building in all its detail.

It is the evolution of the programmable microprocessor that provided the motor for an industry that demands such an investment in men and machines, a continually expanding market is necessary, a market that only the domestic market can satisfy - whether the customer is in need or not. Some of these needs will arise from the possibilities. In education, for example, new techniques, coupled to other technologies such as holography and the video disk with random access, offer for teaching in the home as well as at school an opportunity for a complete transformation of our current educational methods - if there are the teachers capable of exploiting the potentialities. The darker side is the foisting of sophisticated techniques on a public that has no interest in such refined devices as electronically controlled cookers. There is little doubt that despite any purist protests, the march will go on, and the aloof attitude of academic science in Europe only means that products will be made elsewhere, with the unhappy social consequences that the jobs go elsewhere too. Should we not then attempt to control technology or science? Even if we should, we have not yet found the means. 
EPS President, Antonino Zichichi (left) in conversation with Pierre Radvanyi, Chairman of the International Programme Committee.

How does one choose? Take the fascinating developments in the selective multistage photoionisation of molecules by laser beams followed by extraction in the excited state. The first experiments were done over 40 years ago but only now with the advent of a range of tunable and stable lasers can the method be exploited. Selective photoionisation in one application is a powerful means of detecting minute traces of contaminant in an atomic beam - even down to single atoms -in another, it might be used for the separation of uranium isotopes for whatever purpose they are required. At the moment, it is far from clear whether in practice such a system would be economic, but on energy grounds the system looks very attractive. Gas diffusion processes require an energy output of $3.10^{6} \mathrm{eV} /$ atom, electromagnetic separation $10^{6-7}$, the centrifuge $4-5.10^{5}$, ionisation or dissociation by visible or UV radiation $10^{3}$ (assuming a laser efficiency of $1 \%$ ) and dissociation by IR, $10^{2} \mathrm{eV} /$ atom (assuming a laser efficience of $10 \%$ ). On the one hand refined diagnostic techniques and cheaper fuel for nuclear power stations, on the other, the shadow of nuclear war.

\section{The Arms Race}

In the last world war, science was mobilized to serve the military machine, creating institutions that have never been dismantled and like microelectronics take on a momentum of their own. F. Barnaby in a sober and compulsive contribution forced home the solemn realization that $40 \%$ of physicists were engaged on military developments. Dominating the world situation (if not occupying the whole

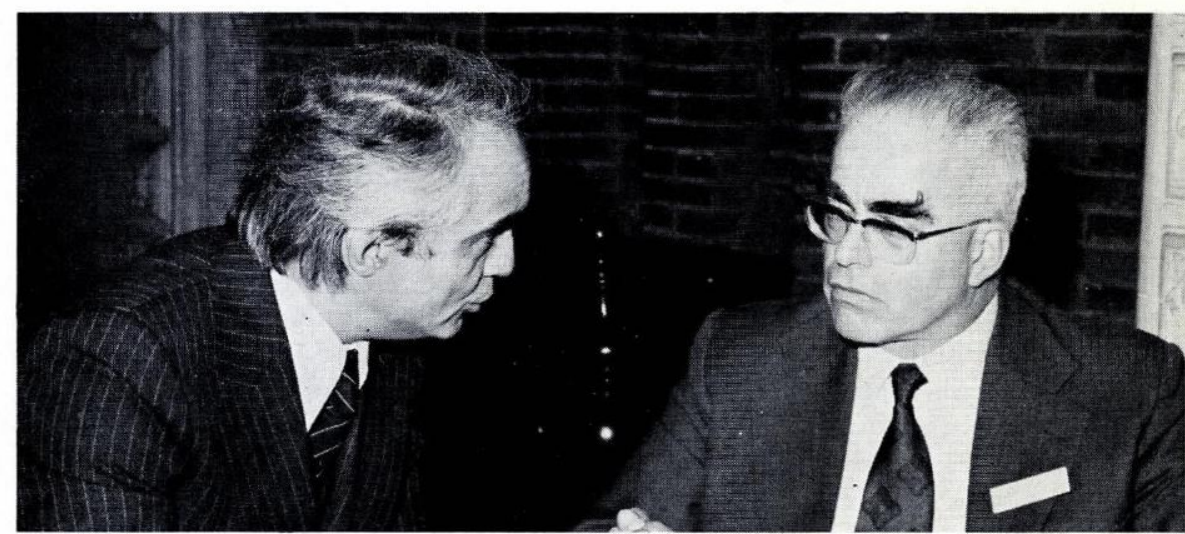

stage) were the nuclear delivery systems deployed by the super powers, an arsenal that amounted to $16000 \mathrm{Mt}$ (TNT) or about 4t (TNT) equivalent for every person in the northern hemisphere. Put another way, all towns of any size are threatened by a fire power equivalent to thousands of the bombs that fell on Hiroshima. Expenditure on weapons has increased linearly from 1960 and is now equivalent to $1 \mathrm{M} \$ / \mathrm{min}$. Over this same period, the number of armed nuclear submarines has increased from near zero to about 250, and some 1400 war satellites have been placed in orbit. The figures are so huge that the mind has difficulty in grasping the enormity of the risk. At one stage, the very grossness of the nuclear weapon seemed to guarantee its exclusion, but 'refinements' in carrying techniques (a modern ICBM weighs $300 \mathrm{~kg}$ and can have an accuracy of $200 \mathrm{~m}$ at $1500 \mathrm{~km}$ ) can introduce the illusion that a nuclear war can be 'won' - at the loss of tens of percent of the population admitted, but short of total annihilation.

Amplifying the risk was the geographical spread of advanced weapon technology, exemplified by a rise in sales to the third world of $3 \%$ to $15 \%$ since 1960. Today, 50 countries produce major weapons systems, about the same number have sophisticated supersonic aircraft and 25 sophisticat-

ed missiles. Any country with about $20 \mathrm{M} \$$ to spare could secretly acquire a few atomic bombs.

The most frightening aspect was the seemingly ineluctable momentum of a military establishment in which the number of scientists far exceeds the number working on disarmament and is even greater than the number working in education. What a desperate waste of human resources ! It was of paramount importance that the SALT talks succeed. Not that these provided a solution, as they tended to cover out-dated or marginally important weapons systems, while technology advanced faster than negotiations. Without agreement however, there was no hope for a start to be made on the real business of disarmament which was the only policy that might save humanity.

But for the physicist as a person, what can he do? Resign from a job that has military funding to go on the dole and see his place taken by some one else ? Barnaby saw the hope, not in personal heroics but in public opinion, in a collective effort to inform the mass of the population of the realities of the situation. Physicists should be in a particularly advantageous position to communicate the facts, and this in turn could lead to pressure being brought to bear in the centres of power. Every physics course should contain information on
H. van Regemorter, Chairman of the Advisory Committee on Physics and Society.

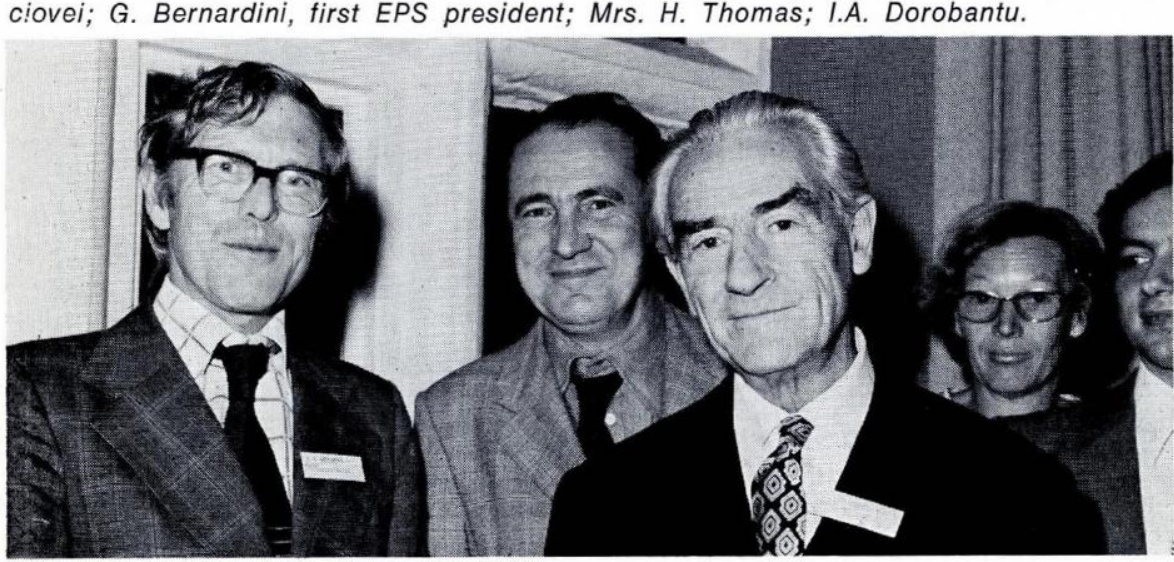

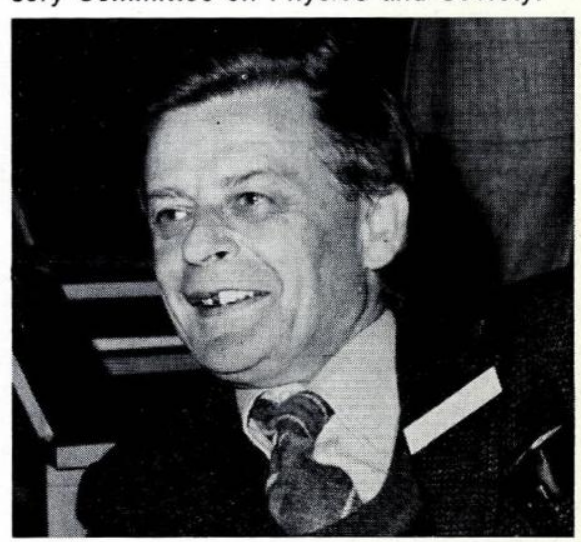


the consequences of technological advance and continued emphasis on military development (which was not the guarantor of jobs often suggested) i.e., of course, if we wished to halt the 'race to oblivion'.

\section{Communication}

Communication is, however, not always the strong point of physicists and to stress the importance of this aspect of our responsabilities, EPS has established a Communications Award that this year has been presented to Sir George Porter "for his distinguished work in the communication of science, and physics in particular, to the public at large, most notably by his Royal Institution lectures, both his evening discourses and the Christmas lectures to children (in London). Through the medium of TV, these have been brought to the attention and have been appreciated by a very large audience."

In acknowledging the award, Sir George paid tribute to his predecessors at the Royal Institution, many of whom had taken great pains to present to an audience comprising leaders of the arts and society and the public at large, the significance of recent advances in science, not scorning the need to provide entertainment at the

\section{Materials Scientists Technical- Scientific University Tehran}

The University is seeking additional faculty members in the various fields of physical metallurgy for the expansion of its Metallurgical Engineering Department. Candidates should have a Ph.D. and active interest in the current development of research programmes in order to supervise M.Sc. and Ph.D. thesis projects. Appointments will be made at the assistant professor level or higher, depending on qualifications and experience.

Please send curriculum vitae, list of publications, and letters of reference to :

Dr. F. Djadali,

Technical- Scientific University, Imperial Iranian Armed Forces, P.O. Box 837,

Tehran, Iran. same time. Evoking the names of Sir Humphry Davy (whose bicentenary is celebrated this year), a noted European (if not EPS) Lecturer and poet, his 'greatest discovery' - Faraday, Tyndall, Dewar, Bragg, he reminded his listeners that popularization, far from being associated with a lowering of standards, was in former days considered a natural concomitant of stature. Some physicists did not have the necessary talent; that had to be recognized, but it was a weakness that gave no cause for arrogance. A good formula to adopt was a little bit of instruction and a lot of entertainment.

Communication has also been occupying a Study Group of the Physics and Society Committee which saw the necessity to help people distinguish between science, applied science and technology. Decisions resulting from scientific discoveries were not the prerogative only of scientists but the scientist was in the best position to inform. One had to be clear also what the objectives were, simply to inform? defend physics? teach physics?. These all demanded a different approach. It may be effective with governments and industrialists to parade the technological results of scientific research, but the same approach to a general public could be disastrous. Physicists had to use their initiative and adaptability to pursue new means of communicating with the public, both directly and through the media, and use the internationalism of science to foster exchanges between different countries.

\section{Developing Countries}

A sharp reminder that the world did not end with the technologically advanced countries came during the Cecil Powell Memorial Lecture given by Prof. M.G.K. Menon, Secretary to the Government of India's Department of Electronics. There were inherent dangers in classifying countries into developed and developing as within each group there was a very large range of wealth, not only measured by per capita income. One could, nevertheless, identify the large number of countries where much of the population lived below the poverty line. Out of a world population of $4000 \mathrm{M}$, $570 \mathrm{M}$ lived below the nutritional level, $800 \mathrm{M}$ were illiterate, $1000 \mathrm{M}$ had inadequate housing and $1500 \mathrm{M}$ inadequate health care.

A lot had been learned in the past 30 years about help to the developing areas, in particular the lesson that the blind importation of technology created as many problems as it solved. There have to be structures inside the country to take advantage of technological advance. The financial aid schemes of the 50's and 60 's led inevitably to massive debts amounting to $100 \mathrm{G} \$$ to the point that debt serving took priority over growth. The last decade had seen a change in this as debt levels have been brought down to those common in developed countries. Massive efforts have been made to develop an infrastructure, widen education and skills and, with one sixth of the capital input considered at one time to be necessary, a growth of twice the estimated had been achieved.

The key lay in collective self-reliance - a moral perhaps for all emerging societies, including the newcomers in the developed areas (such as EPS). Technology should not just be bought on the world's supermarkets. Although in the developed countries, it was accepted that wealth was a direct consequence of science and technology no one really understood the connexions. In the past, in these countries, wealth came from capital investment producing increased productivity per capita. In the developing countries, it was more important to create jobs than goods. It was necessary also to consider the quality of life and not plunge into the race to satisfy needs that have been artificially generated.

This was an argument in favour of fundamental research which formed part of a country's culture. India took pride in its cylindrical radio telescope at the Tata Institute, $35 \mathrm{~m}$ across and $530 \mathrm{~m}$ long that had been set up for interferometry work and had stimulated such competence in antenna arrays and associated electronics that it had been possible to launch an experimental programme of education in rural areas by TV satellite links. It is now proposed to build a $50 \mathrm{~m} \times 2 \mathrm{~km}$ radiotelescope for lunar occultation studies which would be the largest in the world in $\mathrm{m}$ and $\mathrm{dm}$ wavelengths, and would attract to India top experts from all over the globe.

Nevertheless, physics in the developing countries had to be linked closely to society's identified needs and to industry, where an understanding of the laws of nature, and the application of the scientific method were vital. In applied science it was equally vital to understand where the real frontiers of knowledge were, so that applied research could be efficiently planned.

How could physicists in the developed countries help? The P. \& S. 
Participants in the central conference hall at a plenary lecture.

Working Group considered that real care had to be taken to study the individual needs of each area and its particular cultural background. Of general application was the principle of establishing personal contacts, holding conferences in the developing areas and above all treating the physicists from these areas as peers. Redundant equipment might find a use there, but only if it was robust and in full working order. Beware of the dangers of exporting second-hand science.

\section{Quantity vs Quality}

Real progress had been made, at least in India, in demographic control which was of crucial importance if increased wealth was not to be spread even more thinly. Challenged to estimate the signifiance in energy production terms of recent work in which photosynthesis had been artifically imitated (page 12) H.T. Witt retorted that it was better to limit demand than try always to produce more. The quality of life had a tendency to be inversely proportional to quantity. In practical terms though it was essentially an engineering question rather than physics. The process itself was inherently efficient (@70\%) even if in energy production terms the natural cycle was highly inefficient, but this did not say that in a practical plant the energy investment in such prosaic functions as membrane cleaning would not exceed the energy output. It was perhaps more valuable to look for better trees, or train microorganisms, than try to synthesize what Nature did quite successfully already.

\section{Spectroscopy and Synchrotron Radiation}

Standing out from the physics at York, one saw the emergence of optics and spectroscopy. To quote S. Kapitza, 'it used to be said that physics consisted of physics and spectroscopy; this was no longer true'. Spectroscopy was invading all sectors and becoming the most important technique for solving problems in all sorts of fields. Lasers and now synchrotron radiation, providing powerful and complementary sources over such a large frequency range, were opening a whole new range of exploration and if one could design 'wigglers' or 'undulators' in storage rings that would produce coherent synchrotron radiation, such a source would be even more dramatic as a

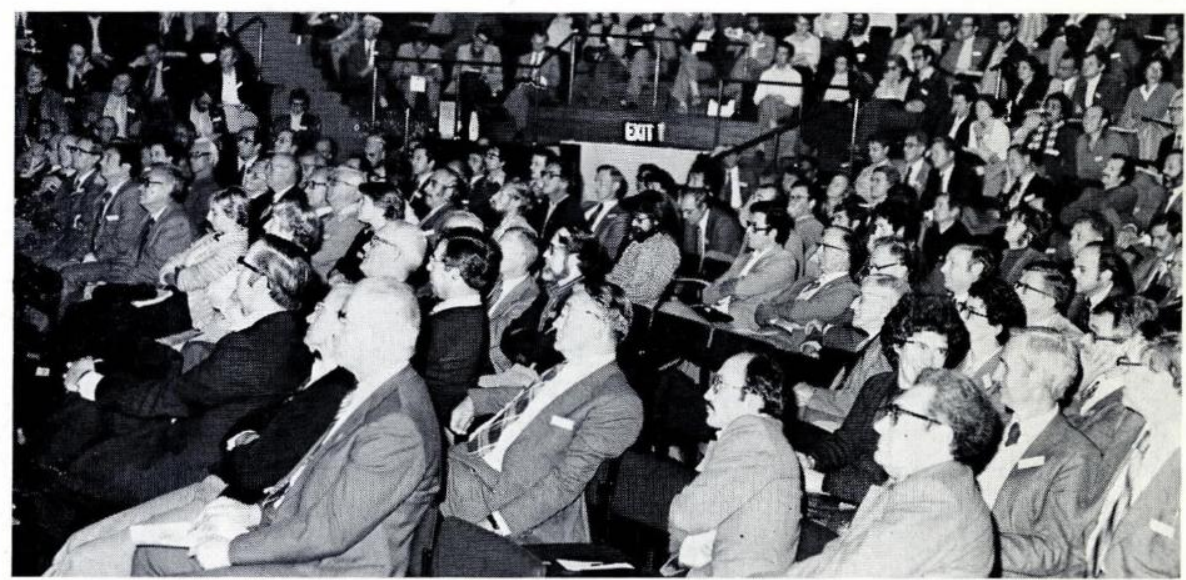

probe. As it was, synchrotron radiation provides a source of photons of high intensity, the energy of which can be chosen. The beam is practically as directional as a laser, is polarized and pulsed so that a 100 ps pulse can be separated by a gap of 100 ns.

Synchrotron radiation spectroscopy was in consequence spreading far outside the field of physics. At the Daresbury accelerator for example, there were over 100 groups clustered round the machine from many different fields. The disciplines of big machine science as known in the high energy physics field would have to be learned by the biologists and chemists too.

An example of the new range of measurements that can be made was the photoemission from surfaces leading to a picture of the detailed electronic structure of solid insulators. Previously we had been limited to making Fermi surface studies of conductors but there was now enough energy in the photons to lift the electrons clear. Resolution was not as good as the Fermi measurements, but good enough. Different aspects of the molecular structure can be studied where the symmetry of the system is very different from that found in atomic physics. Now we can do cross beam experiments looking at the electrons as they come out of a photoionization event and watch the change as molecules break up; also coincidence experiments can be made between ionic fragments and emitted electrons to look at spatial distributions following a photon-molecule collision. Speed of measurement is often restricted by detector saturation but even so, using white-beam X-ray energy dispersive methods, a team at DORIS has obtained reliable powder patterns from $0.2 \mathrm{~mm}^{3}$ samples in $1 \mathrm{~s}$.

\section{Experimental and Theoretical Physicists Technical - Scientific University Tehran}

Applications are invited from experimental and theoretical physicists for visiting faculty positions in the Faculty of Applied Physics. Candidates should have a Ph.D. and an active interest in current research. Duties will be equaly divided between research and teaching at both graduate and undergraduate levels. Candidates will also be expected to supervise M.Sc. and Ph.D. theses.

Appointments will be made at the assistant professor level or higher, depending on qualifications and experience.

\footnotetext{
Applications may be made in the following areas :

amorphous and small gap semiconductor physics.

physics of solar energy.

atomic and molecular physics including laser spectroscopy.

theoretical (nuclear, atomic and molecular, solid state, and high energy) physics.

applied mathematics.
}

Please send curriculum vitae, list of publications, names and/or several letters of reference to :

Dr. F. Djadali

Technical- Scientific University, Imperial Iranian Armed Forces,

P.O. Box 837, Tehran, Iran. 


\section{Astrophysics}

Does synchrotron radiation provide the explanation for cosmic rays which seem to come from supernovae or remnants of surrounding pulsars? The Fermi theory postulated that particles were accelerated by interaction with shock fronts moving in interstellar space, but recent measurements indicate that the plasma temperature is too high to produce isotropic radiation and the solution might reside in the particles being picked up by synchrotron radiation emitted by a pulsar where the magnetic axis is inclined to the rotational axis. Supporting evidence comes from the fact that the rate at which the pulsar in the Crab nebula looses energy by spin-down, is comparable with the rate at which the nebula loses energy in the form of radiation.

The astrophysicists can always astonish in the range of dimensions they grapple with. In the sun, it is generally agreed that energy storage is in the magnetic field in the neighbourhood of a sunspot - a force free field where the currents are parallel to the field lines. After a flare, during which some $10^{32}$ erg can be released in a few seconds, the field relaxes to a minimum energy condition with no current flow, but how? One proposal is that the change is brought about by a tearing mode instability where the current sheets break up into filaments. Fine, were it not that the sheets would have to be only $1 \mathrm{~cm}$ thick.

In the past we have constructed a pretty satisfactory picture of the build up of elements in the solar system, although it seems that recent measurements of the $\mathrm{O}_{2} / \mathrm{N}_{2}$ ratio in the $\mathrm{H}$ II regions of the galaxy lead to the conclusion that there is much more internal mixing than once thought. Now the art is to identify the sites where the various nuclear processes go on. Behind these processes however lies the basic question as to the source of protons. According to Dirac, when the atomic number of a nucleus $Z \cong 170$, the binding energy of the inner electrons should exceed twice the electron rest mass and pair production should occur, as heavy ion experiments of uranium on uranium have demonstrated. Similarly, if the gravitational energy is sufficiently high as in a black hole, pair production takes place and one particle can emerge. Going further, it can be postulated that this was the source of matter in the Universe - matter being created from a disembodied gravitational field - but the excess of matter over anti-matter is still bothering.

\section{Super Spin and Mass}

Heavy ion physics is currently one of the most promising branches of physics as the new heavy ion accellerators come into operation. These are able not only to surmount the Coulomb barrier of heavier elements but impart so much spin, the deformation modes of nuclei can be studied, stimulating the creation of new models of nuclear structure. With very high spin and what is effectively a low temperature as the energy is ordered into one degree of freedom, a phenomenum akin to super conductivity has been observed and the reason is probably similar; viz. a result of nucleons binding into pairs in the same way that electrons do in super-conducting materials.

It seems that there is little hope now of finding superheavy elements in Nature, although theory insists on predicting these islands of stabilty where nuclei would have a half life longer than that of the earth. It is up to the big machines to provide the answers.

\section{Fusion}

A category of machine that continues to receive much attention is the Tokamak and one is obliged to acknowledge that in plasma physics

Central area of the exhibition mounted in the physics buildings.

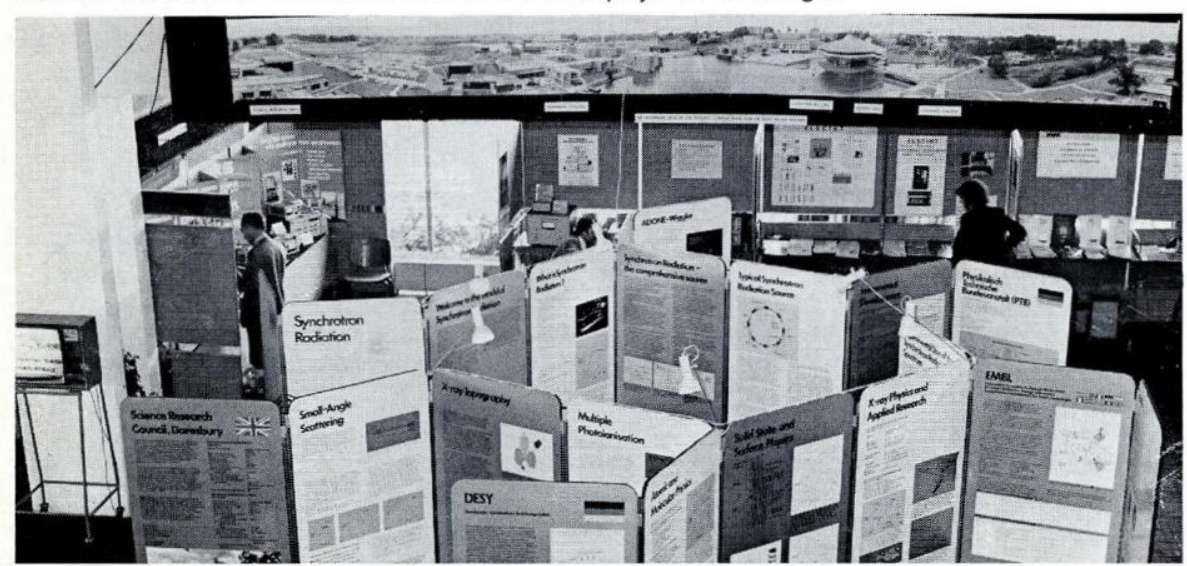

the islands are in our understanding, surrounded by a great deal of uncertainly. Simple theories do not suffice. For example, only if electronplasma interactions are considered relativistically do we begin to expect the turbulence that is encountered. Our assumptions of isotropy and homogeneity are misleading and it is probable that as theory does not take account of magnetic fields, which in practice are not negligible, it is perhaps not surprising that the theoretical picture is incomplete. With only one order parameter, however, it is difficult to do better. Magneto-hydrodynamics is much more complicated than quantum mechanics where the eigen value enters in a simple way. Spectra are more complicated and it is now concluded that there are two continua with five discrete spectra on both sides.

In the toroidal situation, the essential parameters are the ratio of kinetic to magnetic pressure, $\beta$, and the security factor $Q$ which is linked to the current. There is no equilibrium state beyond an upper value of $\beta$ but the higher its value, the greater is the efficiency in using the magnetic field. One conclusion is that the plasma should have a D - shaped cross section, elongated vertically - so that contrary to the normal laws of life it seems that engineering decisions taken for JET are in line with the requirements of the physics. $Q$ is improved as well as the $\beta$ limit if there is a current flowing round the plasma in the toroidal direction but how long this can be sustained is unkown.

One cheering discovery was the fact that cyclotron radiation from the plasma could be used as an on-line diagnostic probe. The frequency of the radiation emited by the electrons spiralling round the magnetic field depends only on the field, while the intensity gives information on the temperature and density. The advantage over laser beam scattering is that information is given for every machine shot and does not have to be scanned over a series, during which conditions can change.

\section{Velocity of Light}

As an interdisciplinary exercise, the modern optics symposium was highly successful (see below) but one further point deserves emphasis, notably the prospect of deriving a continuous set of interrelations from X-rays through to the VUV. Central to this is the velocity of light which is now known to an accuracy two orders of magnitude better than previously :

$c=299792458 \pm 1.2 \mathrm{~m} / \mathrm{s}$. 